\title{
Amyloid PET in the diagnostic workup of neurodegenerative disease
}

\author{
Pierpaolo Alongi ${ }^{1} \cdot$ Agostino Chiaravalloti $^{2,3}$. Valentina Berti ${ }^{4} \cdot$ Cecilia Vellani $^{5} \cdot$ Giuseppe Trifirò $^{5} \cdot$ Giulia Puccini $^{6}$. \\ Giulia Carli ${ }^{7} \cdot$ Andrea Chincarini $^{8} \cdot$ Silvia Morbelli $^{9,10} \cdot$ Daniela Perani $^{7} \cdot$ Stelvio Sestini ${ }^{6}$
}

Received: 28 January 2021 / Accepted: 27 April 2021 / Published online: 23 June 2021

(C) Italian Association of Nuclear Medicine and Molecular Imaging 2021

\begin{abstract}
Several radiotracers have been employed to bind amyloid plaques in the brain for the differential diagnosis of neurodegenerative disease, open the possibility to measure in vivo pathogenic processes implicated in Alzheimer disease (AD) and have the potential to detect disease processes at the earliest stages with a potential role in clinical trials' longitudinal assessment. Visual reading is clinically adequate in clearly positive or negative cases but the adoption of validated and standardized quantification methods of amyloid-PET to diagnose AD in research and clinical settings has become essential. The present Pictorial Essay presenting a case series of nine patients describes the current role of amy-PET imaging elucidating the typical assessment, the possible artifacts and pitfalls occurring in the clinical scenario, focusing also on the development of quantification methods in the diagnostic workup of neurodegenerative disease.
\end{abstract}

Keywords Positron emission tomography computed tomography $\cdot$ Amyloid PET $\cdot$ Alzheimer's disease $\cdot 18 \mathrm{~F}$-Florbetaben . 18F-Florbetapir · 18F-Flutemetamol

Stelvio Sestini

stelviosestini@gmail.com

1 Nuclear Medicine Unit, Fondazione Istituto G. Giglio, Cefalù, Italy

2 Department of Biomedicine and Prevention, University of Rome Tor Vergata, Rome, Italy

3 IRCCS Neuromed, Pozzilli, IT, Italy

4 Department of Biomedical, Experimental and Clinical Sciences "Mario Serio", University of Florence, Florence, Italy

5 Nuclear Medicine, ICS Maugeri SpA SB-IRCCS, Pavia, Italy

6 Nuclear Medicine Unit, Nuovo Ospedale S. Stefano of Prato, Via suor Niccolina Infermiera 20, Prato, Italy

7 Division of Neuroscience, Vita-Salute San Raffaele University, Milan, Italy

8 Istituto Nazionale di Fisica Nucleare (INFN), Genoa, Italy

9 IRCCS Ospedale Policlinico San Martino, Genoa, Italy

10 Nuclear Medicine Unit, Department of Health Sciences, University of Genoa, Genoa, Italy

\section{Introduction}

Early diagnosis of neurodegenerative dementia as well as reliable etiological diagnosis of the causes underlying cognitive decline remains challenging on the basis of merely clinical assessment.

Biomarkers are increasingly used with a Spectrum of available modalities [magnetic resonance imaging (MRI), positron emission tomography (PET), cerebrospinal fluid (CSF), upcoming blood biomarkers]. While the appropriate combination and/or order in which these biomarkers should be used in diagnostic evaluations is still partially debated, the International Working Group-2 (IWG-2) criteria [1], and more recently, by the last National Institute of AgingAlzheimer Association (NIA-AA) [2-4], measurement of amyloid-beta precursor protein $(\mathrm{A} \beta)$ deposition in the brain using PET imaging is a suitable strategy to specifically support the suspect of dementia (or mild cognitive impairment) due to Alzheimer's disease (AD).

Several PET radiotracers have been validated for imaging of amyloid deposition (amy-PET) in the brain (namely, 11C-PIB, 18F-Florbetaben, 18F-Florbetapir, 18F-Flutemetamol). These tracers have been demonstrated high accuracy to support the clinical differential diagnosis between $\mathrm{AD}$ vs non-AD dementia [5, 6]. Measurable impact in terms of 
patient management and final diagnosis changes has been demonstrated [7, 8]. Of note, amy-PET scans specificity for the identification of brain amyloidosis due to AD might be partially hampered in patients older subjects (i.e. $>75$ years) due to possible incidental brain amyloidosis as well as in patients with other diseases (i.e., in patients' with amyloid angiopathy or in patients with other neurodegenerative diseases such as dementia with Lewy bodies in which brain amyloid has been reported).

Although visual reading is clinically adequate in clearly positive or negative cases, it is in a non-negligible fraction of cases the presence of a positive or negative amy-PET cannot be easily captured using only visual evaluation [9]. Semiquantitative assessment can better identify borderline cases and support the nuclear medicine physician in the informed writing of the clinical report.

Thus, the adoption of validated and standardized quantification methods of amy-PET to accurately define the presence of brain amyloidosis in research and clinical settings has become essential. Different approaches have been proposed for semi-quantitative analysis of amy-PET imaging [9-11].

In this Pictorial Essay, we show the current role of amyPET imaging and semi-quantification methods in the diagnostic workup of neurodegenerative disease, emphasizing both clinical pitfalls and technical artifacts may affect images assessment. Each one of the presented cases aims to provide evidence of the potential added value and drawbacks of amy-PET imaging when used in the currently accepted state-of-the art algorithm [12,13]. Accordingly, structural MRI imaging is proposed as a first step (following clinical and neuropsychological evaluation), as it allows detecting other potential pathologies that could be responsible for cognitive decline and can allow the preliminary identification of topographical patterns of atrophy. FDG PET data are included (before amy-PET studies) when appropriate as can support early diagnosis showing the AD characteristic patterns of neurodegeneration at the MCI stage (earlier than MRI). Finally, added value of amy-PET is here underlined for its capability to reliably establish the etiological diagnosis of AD in patients, including in the clinically atypical variants of $\mathrm{AD}$. The value of a negative amyloid-scan for the exclusion of $\mathrm{AD}$ in patients with for cognitive impairment is also illustrated.

\section{Materials and methods}

A clinical case series aiming to highlight the current role and pitfalls of amy-PET imaging in the diagnostic workup of neurodegenerative disease was retrieved in the framework of multicentre Italian collaboration within the AIMN Working group of Neurology.
Nine clinical cases describing optimal scan acquisition and image interpretation, the typical and early AD presentation, artifacts, and pitfalls in the clinical practice using amyPET imaging are reported. Methods for semi-quantification of amy-PET imaging in part of the cases are also presented and briefly discussed. In all cases, images were acquired following SNMMI/EANM joint guidelines [14].

The following clinical, biochemical, and further imaging features have been reported when available: age, scholarly, neuropsychological evaluation, cognitive performance (MMSE, CDR), CSF (beta-amy, Tau, p-Tau levels), MRI, $\left[{ }^{18}\right.$ F]Fluorodeoxyglucose (FDG) PET imaging.

\section{Quantification and imaging analysis methods}

The relative quantification (or semi-quantification) in amyPET is generally an expression of the tracer uptake ratio between regions of interest (ROI), consisting in the average intensity computed in one (or more) uptake ROI divided by the average intensity in a reference ROI; this procedure defines the SUVr. For its straightforward implementation, SUVr is the most widely used method in clinical practice. However, this measure is plagued by some limitations mostly due to the variability linked to the tracer, the uncertainties in the segmentation of the uptake and reference ROI, and the possible choice of the ROI.

To improve the stability, and therefore, the clinical applicability of the semi-quantification other SUVr-independent methods have been developed. In this essay, we show a case that underwent three semi-quantification evaluations: a cortical-cerebellar SUVr, the ELBA method (Evaluation of Brain Amyloidosis) and the TDr method (Time-Delay ratio). In addition, all these have been evaluated on a regional atlas derived from the AAL and consisting of 25 cortical contralateral ROIs.

ELBA is not based on the uptake or reference ROIs and does not compute the ratio between counts. It rather relies on gradients of uptake along iso-curves departing from the high uptake in the white matter. TDr is more like SUVr but without predefined uptake and reference ROIs, these being extracted from the early perfusion scan. All these three methods have advantages and limitations. ELBA results have been previously demonstrated to be possibly affected by scan quality [15]. In TDr validation study, TDr and ELBA results demonstrated a closer correlation on the whole population and on the classification of negative and positive subjects. This could be ascribed to the inherent SUVr variability due to the fact that uptake and reference ROIs are predefined in size and positioning, which can lead to larger errors on a single subject.

Together, these three methods provide independent approaches to semi-quantification which rely on different information present in the PET scan. Ideally, a ranking 
system based on more than one method and incorporating visual reading ass well might result in a drastic reduction of the semi-quantification uncertainty and allow the clinician to properly evaluate and discuss borderline cases [16]. Finally, it should be noted that in clinical practice, amy-PET is generally globally inspected to provide a binary outcome. However, evaluation of regional amyloid load might have a further added value. Indeed, in the research settings, quantification methods highlighted different susceptibility to $A \beta$ accumulation among brain regions while the association between cognition and $\mathrm{A} \beta$ distribution is still under investigation [17].

Here, we present two clinical cases where quantification using three different quantification methods for global amyloid load have been adopted. Regional $\mathrm{A} \beta$ assessment was not performed but it might represent an available resource for the analysis of amy-PET scans with potential clinical and prognostic in the next future.

\section{Optimal scan acquisition and image interpretation}

\section{Case \#1: amy-PET with 18F-Flutemetamol}

A female patient in the 5th decade of life with amnestic Mild Cognitive Impairment (MCI)-single Domain MMSE 28/30 with a family history of $\mathrm{AD}$, under follow-up for anxiousdepressive disorder for years. The patient underwent multiple MRI scans showing no significant alterations. Then, the patient performed a 18F FDG brain PET (Fig. 1a). Transaxial slices were re-orientated to fit, respectively, the bicommissural line (A) and the major axis of temporal lobes (B). The visual analysis of the brain scan showed hypometabolism in bilateral temporo-medial and parietal cortices without a significant posterior cingulate gyrus and precuneus involvement. Significant hypometabolism in temporal regions as compared to controls was confirmed using

A
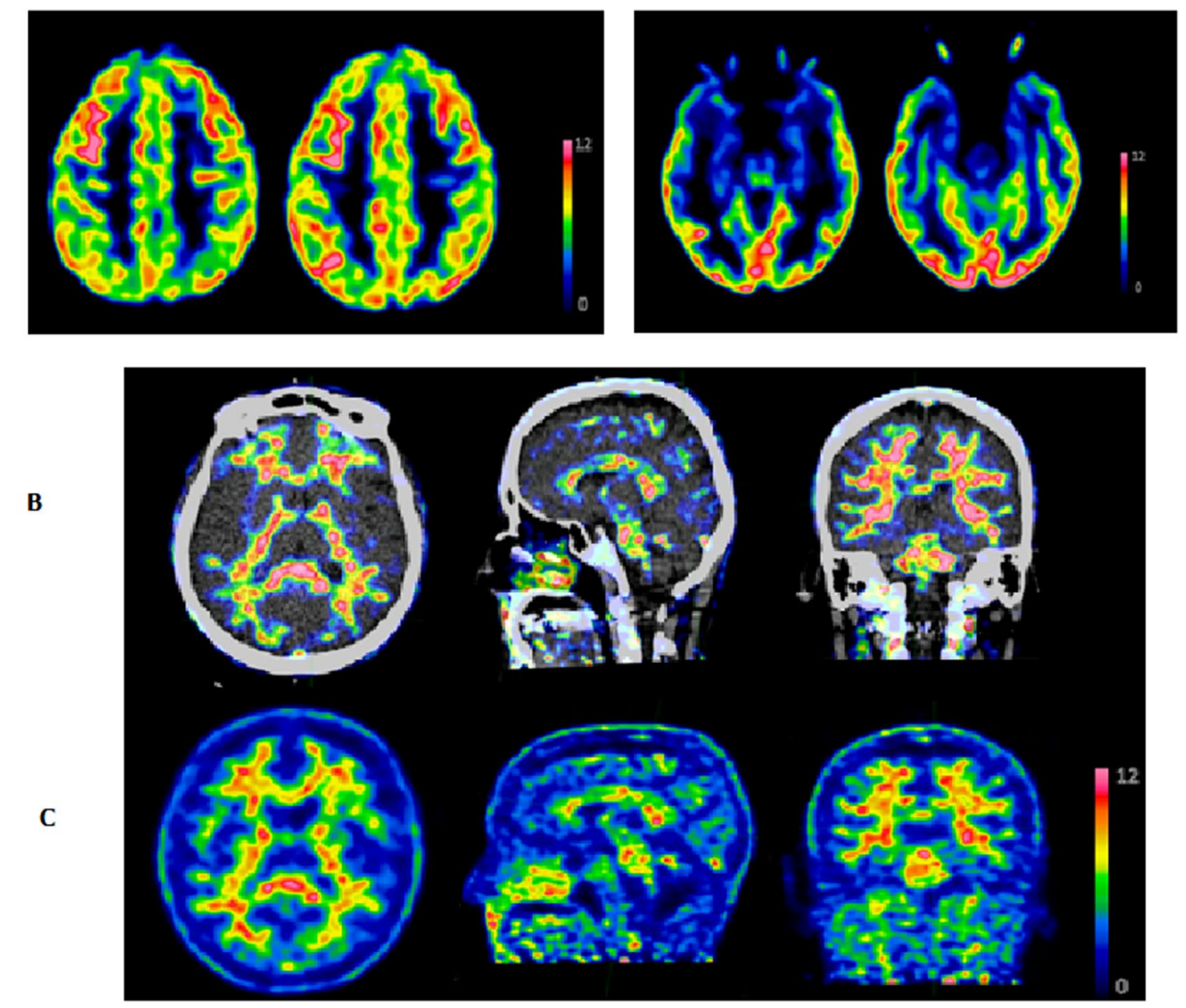

Fig. 1 Case \#1-optimal scan acquisition and image interpretation with 18F-Flutemetamol: 18F FDG brain PET images A and amy-PET with 18 F-Flutemetamol $(\mathbf{b}, \mathbf{c})$ axial, sagittal and coronal brain slices on Spectrum color scale are displayed, respectively, without (bottom) and with (top) co-registered low-dose CT 
a semi-quantitative approach with a Single subject Voxelbased SPM analysis ( $p$ 0.05).

Subsequently, amy-PET with $18 \mathrm{~F}$-Flutemetamol was performed to estimate B-amyloid neuritic plaque density in the cerebral cortex (Fig. 1b, c). Axial, sagittal and coronal brain slices on Spectrum color scale are displayed, respectively, without (bottom) and with (top) co-registered low-dose CT to evaluate brain atrophy. The amy-PET images were visually analyzed systematically, starting at the level of pons and scrolling up through cortical and sub-cortical gray matter, including the frontal, temporal and parietal lobes, posterior cingulate, precuneus and striatal region. Interpretation of the amy-PET images was performed by comparing the activity in cortical gray matter with activity in adjacent cortical white matter. The images showed a sulcal/gyral white matter pattern and a tapered intensity to the tissue's periphery. The intensity of tracer signal in cortical regions was pretty lower compared with adjacent white matter and similar in intensity to the gray matter-rich regions of the cerebellum, suggesting a negative flutemetamol scan. The interpretation of amy-PET images was based on visual reading and confirmed by quantification analysis of the amyloid burden in different brain regions [18].

\section{Case \#2: amy-PET with 18F-Florbetaben}

A female patient in the 5th decade of life with familiar positivity for neurodegenerative disease and neurological assessment underlining progressive short-term memory deficits, inattention in daily life, the tendency to excessive spending, occasional episodes of disorientation, hypovisus, and resting, postural and kinetic tremor. The patient showed a lack of awareness of cognitive disorders, difficulties in using the telephone, easy irritability and mild repetitive behaviors. The functional neurological and cognitive scores were: Instrumental Activities of Daily Living (IADL): 0/8, Basic Activities of Daily Living (BADL):2/6, Mini-Mental-State Examination (MMSE) 23/30, CDR 1. Eco-Color Doppler of supra-aortic vessels demonstrate diffuse medio-intimal thickening. On the right, presence on the posterior bulbar wall and in the I section of the ICA of a fibro-lipid plaque with a regular surface (maximum thickness $0.20 \mathrm{~cm}$ ), not hemodynamically significant (VPS $\max 0.64 \mathrm{~m} / \mathrm{s}$ ).

Brain Magnetic resonance imaging (MRI) showed signs of chronic vascular distress of the peri-ventricular white matter and radiated crowns; diffuse slight atrophic enlargement of the supratentorial ventricular system and of the pericerebellar and peri-encephalic liquor spaces of the base and vault, more evident in the fronto-parietal site at the convexity (Fig. 2d-f).

Subsequently, the neurologist requested a Brain 18F FDG PET/CT demonstrating hypometabolism mainly affecting the posterior encephalic structures in the bilateral parietooccipital area (Fig. 2g-i).

The patient started therapy with Donepezil as suggested by the neurologist, followed by worsening of cognitive disturbances and tremor symptoms.

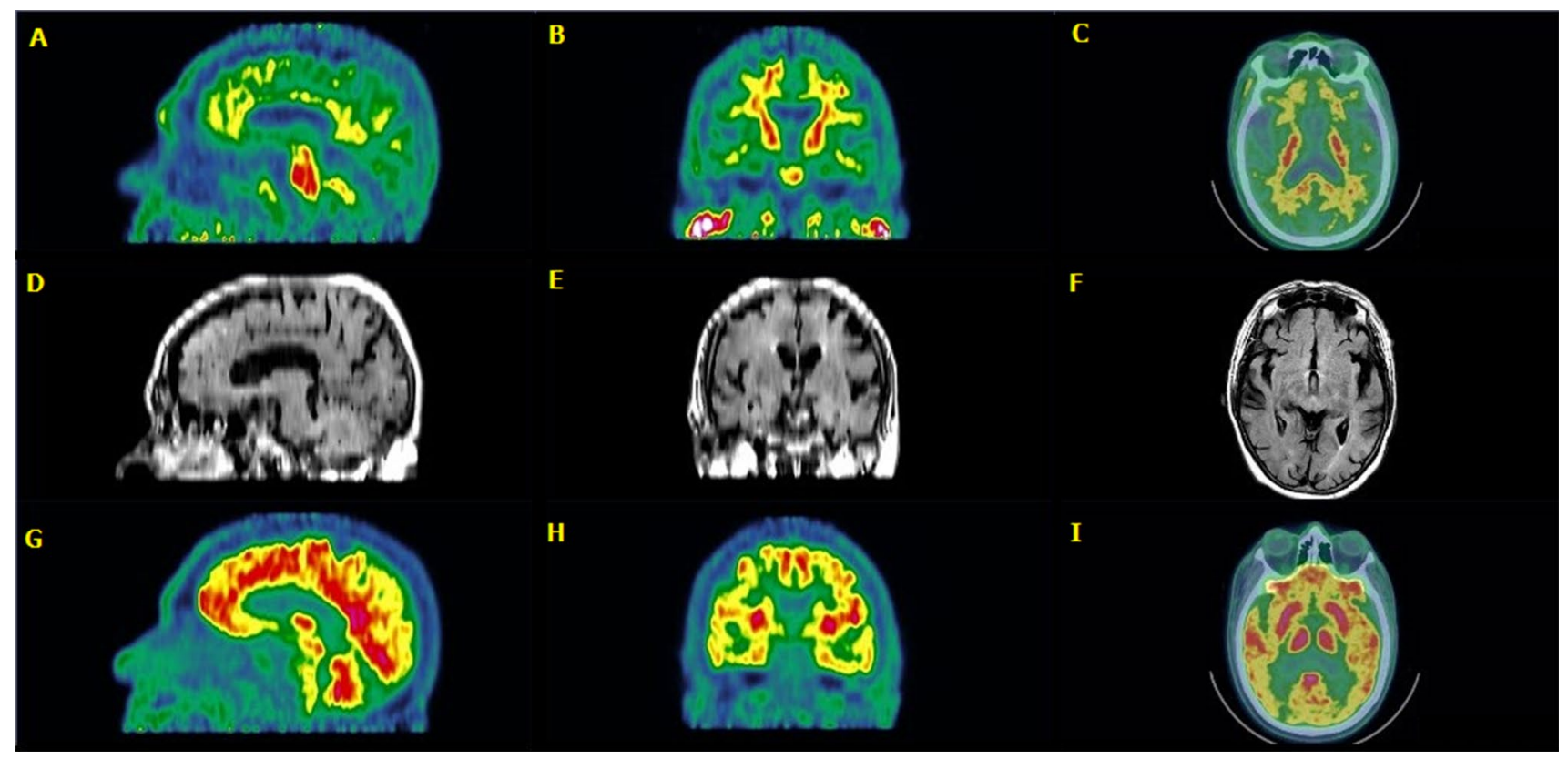

Fig. 2 Case \#2-optimal scan acquisition and image interpretation with 18F-Florbetaben—amy-PET with 18F-Florbetaben (a-c); MRI images $(\mathbf{d}-\mathbf{f})$; $18 \mathrm{~F}$ FDG PET/CT brain images $(\mathbf{g}-\mathbf{i})$ 
Amy-PET with $18 \mathrm{~F}-$-Florbetaben was performed to evaluate the amyloid burden as possible neurodegenerative disease correlate to Alzheimer Disease (Fig. 2a-c). The amy-PET images were visually analyzed as suggested by Neuraceq Training (Life Molecular Imaging Ltd) normalizing on cerebellar peduncles and starting the evaluation through cortical and sub-cortical gray matter including the frontal, temporal and parietal lobes, posterior cingulated and precuneus. Interpretation of the amy-PET images was performed by comparing the radiopharmaceutical uptake in cortical gray matter with the activity in adjacent cortical white matter by selecting the target intensity (maximum intensity on visual assessment) in each region. The images showed that the target intensity corresponds to the white matter in every region, without any significant uptake in the gray matter. The functional uptake in the white matter was well differentiated in retro splenial, temporal and frontal region with a clear recognition of the longitudinal fissure and most of the sulcus/gyrus. The interpretation of the amy-PET images made on visual reading was confirmed by quantification analysis of the amyloid burden in different brain regions. The examination was interpreted as negative for neurodegenerative disease associated with a cortical amyloid burden.

After 1 month, the cerebrospinal fluid assay showed normal $\beta$-amyloid $1-4$ concentration $(1043 \mathrm{pg} / \mathrm{ml}$, normal range 483-3067 pg/ml); therefore, Donepezil was suspended. Patients' follow-up demonstrates no significant worsening of clinical status and floating of symptoms most likely due to vascular brain impairment. Amy-PET demonstrated to be crucial in the workup of patients with neurodegenerative disease, excluding $\mathrm{AD}, \mathrm{AD}$-related amyloid burden and addressing patient's correct management.

\section{Diagnosis of AD with amyloid PET}

\section{Case \#3 typical AD presentation of amy-PET}

A female patient at the end of the 5th decade of life with a neurological diagnosis of Alzheimer's disease. The patient showed an insidious onset and progressively worsening episodic memory deficits for a recent event, with associated semantic-lexical recovery disorders and episodic topographic disorientation. The neuropsychological evaluation showed a significant reduction in the score of the tests performed. Particularly, Mini-Mental State Examination score was 20/30. The test battery showed difficulties in verbal memory tests, in a test of visual-spatial memory, in a test of simple attention, in a test of language on phonological criteria, and a complex constructive praxis test.
MRI showed an enlargement of lateral ventricles, a moderate enlargement of Sylvian fissures and cortical grooves, and convexity without site or side prevalence.

In Fig. 3a, we report the axial, coronal, and sagittal views of amy-PET with 18F-Florbetaben, showing a significant pathological burden of amyloid in the gray matter (frontal, temporal and parietal cortex detailed views are provided of both PET and PET/MRI imaging). In particular, amyloid imaging showed a severe involvement of the associative cortical areas that confirmed the clinical suspicion and helped to clarify the neuropsychological assessment when the outcomes of tests were doubtful. We performed a cerebrospinal fluid assay (CSF) to investigate the possible coexistence of alterations in other CSF parameters as Tau. On this occasion, $\beta$-amyloid 1-4 concentration in CSF was measured to quantify amyloid loads in the brain (the value was $252 \mathrm{pg} /$ $\mathrm{ml}$, normal range $483-3067 \mathrm{pg} / \mathrm{ml}$ ).

In Fig. 3b, we report the axial, coronal, and sagittal views of 18F FDG PET/CT showing a significant reduction of cortical glucose consumption in gray matter frontal, temporal, and parietal cortex bilaterally (detailed views are provided of both PET and PET/MRI imaging).

\section{Case \#4: early diagnosis of AD}

A male patient at the end of the 7th decade of life was referred to a neurologist for short-term memory loss. Neuropsychological assessment demonstrated initial memory, attention, and praxis-constructive deficits. He was also disoriented in time and space and performed poorly in a cued recall test. MMSE 23/30 at the time of referral.

Amy-PET with 18F-Florbetapir was positive for the presence of a significant and diffuse amyloid burden in the brain cortex (Fig. 4). 3T-MRI found moderate signs of leukoaraiosis and cortical atrophy, mainly in the frontal regions (Fig. 5). Liquor analysis was not conclusive as Tau protein was $396 \mathrm{pg} / \mathrm{ml}$ (normal value $<375 \mathrm{pg} / \mathrm{ml}$ ) and beta-amyloid was $570 \mathrm{pg} / \mathrm{ml}$ (normal value $>550 \mathrm{pg} / \mathrm{ml}$ ). After 4 years, the MMSE decreased significantly: 17/30.

In case of a nonconclusive cerebrospinal fluid tau and amyloid results in patients with cognitive decline, amy-PET may help to evaluate the entity of amyloid burden in the brain.

\section{Artifacts on amy-PET images}

\section{Case \#5 positioning/movement artifacts}

A male patient at the end of 5th decade of life was referred to amy-PET for AD/FTD differential diagnosis because of multiple domain cognitive decline (MMSE 20/30). During 


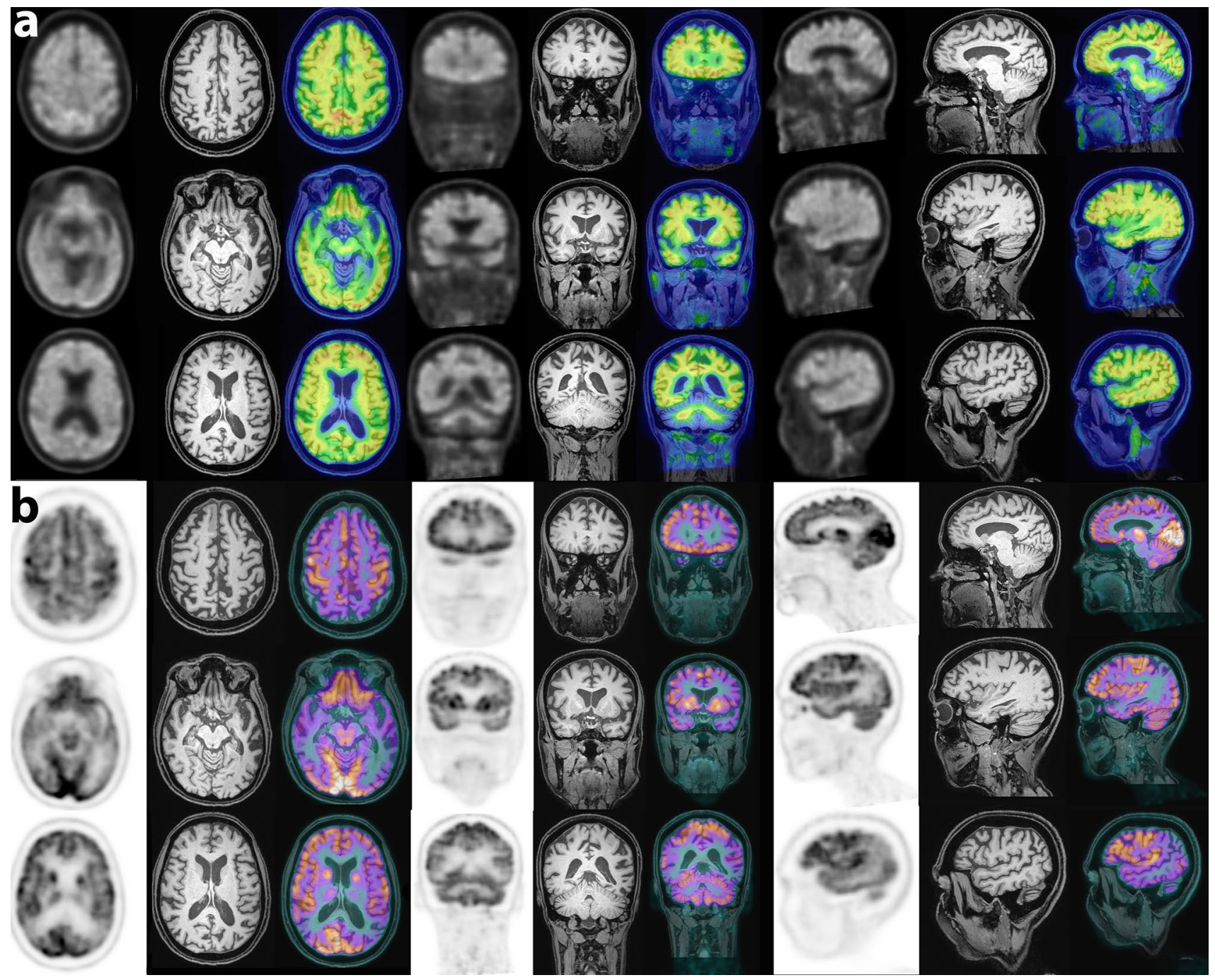

Fig. 3 Case \#3-typical AD presentation of amy-PET: a axial, coronal, and sagittal views of amy-PET with 18 F-Florbetaben, showing a significant pathological burden of amyloid in the gray matter (frontal, temporal and parietal cortex detailed views are provided of both PET and PET/MRI imaging). b axial, coronal, and sagittal views of

amy-PET acquisition patient moved his head on a sagittal plane, causing several artifacts on amy-PET images (Fig. 6).

This case demonstrated the importance of accurately checking the skull's activity and recognizing motion and attenuation correction artifacts before examining gray matter tracer activity.

\section{Case \#6: double skull phenomenon and asymmetries}

A male patient in the 5th decade of life was referred to amy-PET for progressive memory deficits. During PET/
18F FDG PET/CT showing a significant reduction of cortical glucose consumption in gray matter frontal, temporal, and parietal cortex bilaterally (detailed views are provided of both PET and PET/MRI imaging)

CT acquisition, patient moved his head on an axial plane, causing several artifacts on amy-PET images (Fig. 7). The take-home message is to check skull activity in search of double skull phenomenon and asymmetries of activity before examining gray matter tracer activity. If motion happened only between CT and amy-PET acquisitions, a second amy-PET reconstruction should be performed after realignment between amy-PET and CT, if possible. To be able to correct for artifacts due to motion during the amyPET scan, a list mode acquisition should be performed. Alternatively, several frames should be acquired, or a second late acquisition could be performed. 


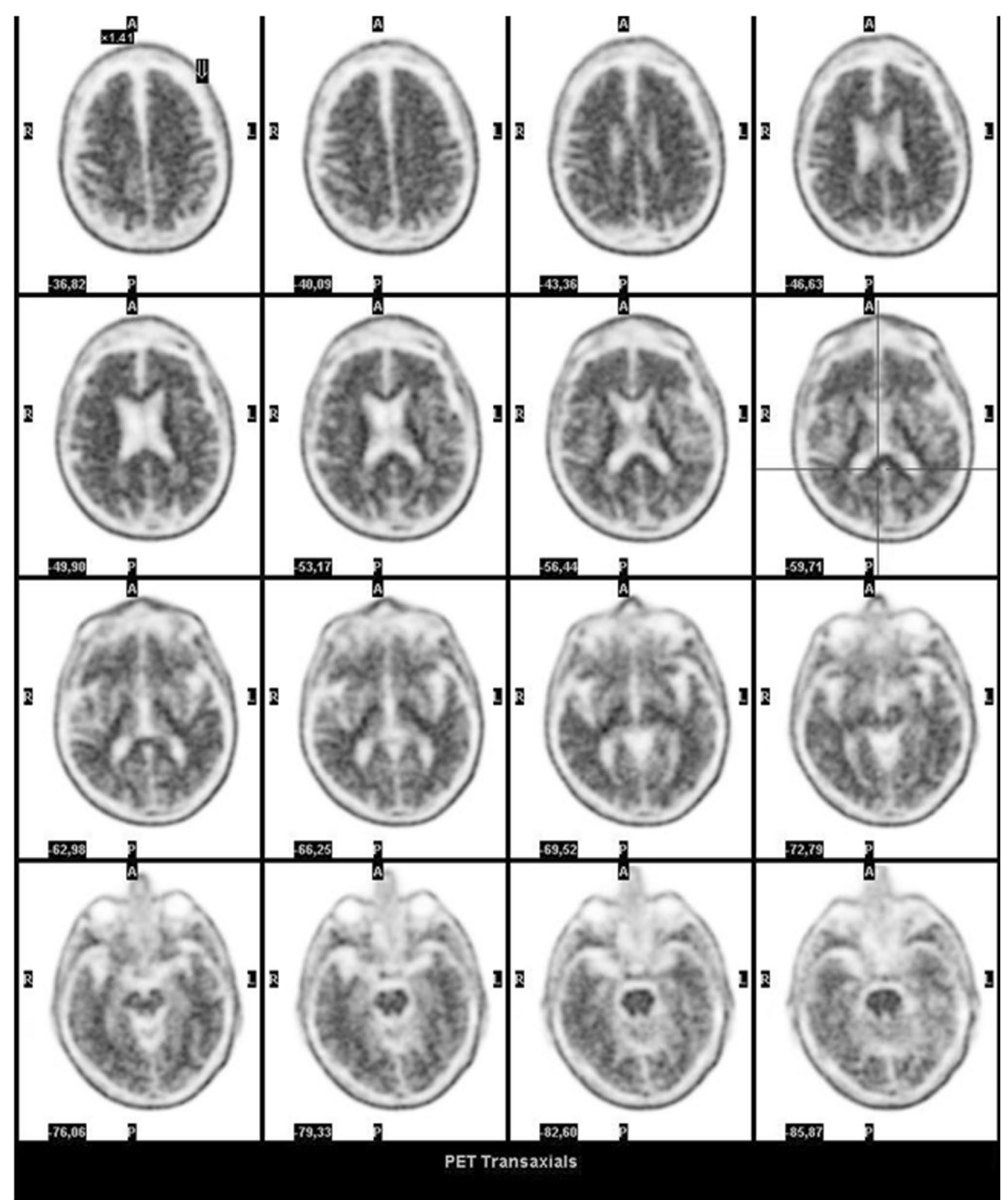

Fig. 4 Case \#4 - early diagnosis of AD: axial amy-PET images with 18F-florbetapir showing significant amyloid burden in the brain cortex 


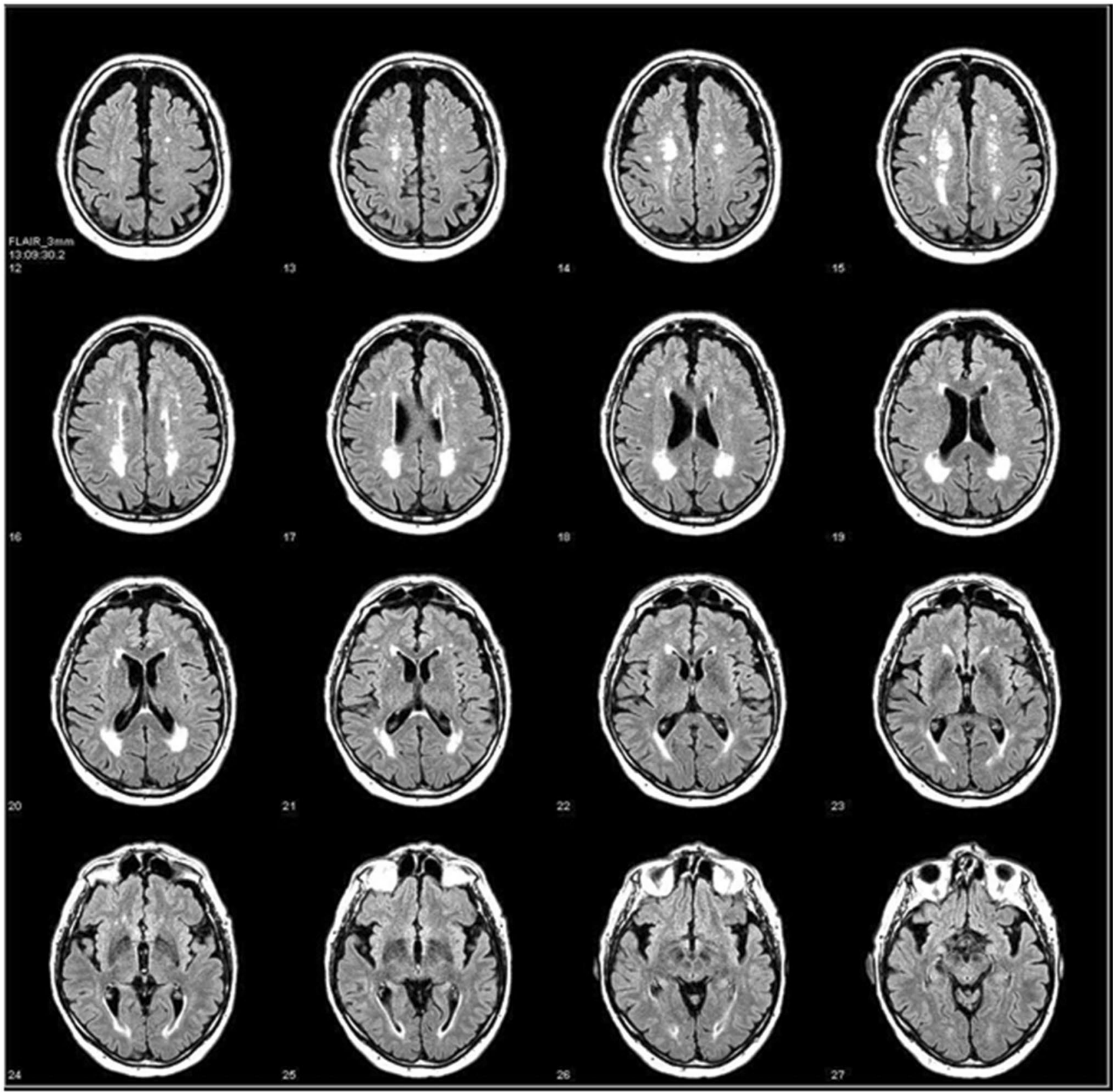

Fig. 5 Case \#4 - early diagnosis of AD: axial 3T-MRI FLAIR images showing moderate signs of leukoaraiosis and cortical atrophy, mainly in the frontal regions

\section{Pitfalls}

\section{Case \#7: positive amy-PET in a patient with cerebral amyloid angiopathy}

A female patient in the 6th decade of life was referred to PET/MR for AD/FTD differential diagnosis because of worsening memory deficits. MR (Fig. 8; T2-weighted TSE on the right column) showed GCA score $=1$, Koedam score $=1$ bilaterally, and MTA score $=2$ on the left and 1 on the right sides. No mesencephalic alterations were noticed. Amy-PET (Fig. 8, left column) demonstrated a clear uptake of 18F-Florbetaben throughout the entire brain cortex consistent with AD. At susceptibility-weighted imaging (SWI), however, a number of little minor, ovoid, cortico-sub-cortical areas of hemorrhage (Fig. 8, red arrows) have been noticed in the left parieto-temporo-occipital cortex that could be consistent with the diagnosis of cerebral amyloid angiopathy. Several T2 TSE hyperintensities in the white matter (Fig. 8, white arrows on the right column) were also 
Fig. 6 Case \#5-positioning/ movement artifacts: first row amy-PET images appear blurry and misalignment between amyPET and CT occurs, mostly visible in frontal lobes. Second row amy-PET artifacts due to patient movement during PET/ $\mathrm{CT}$ acquisition consisted of double skull phenomenon (left) and different activity at opposite sides of the skull and the brain (right)

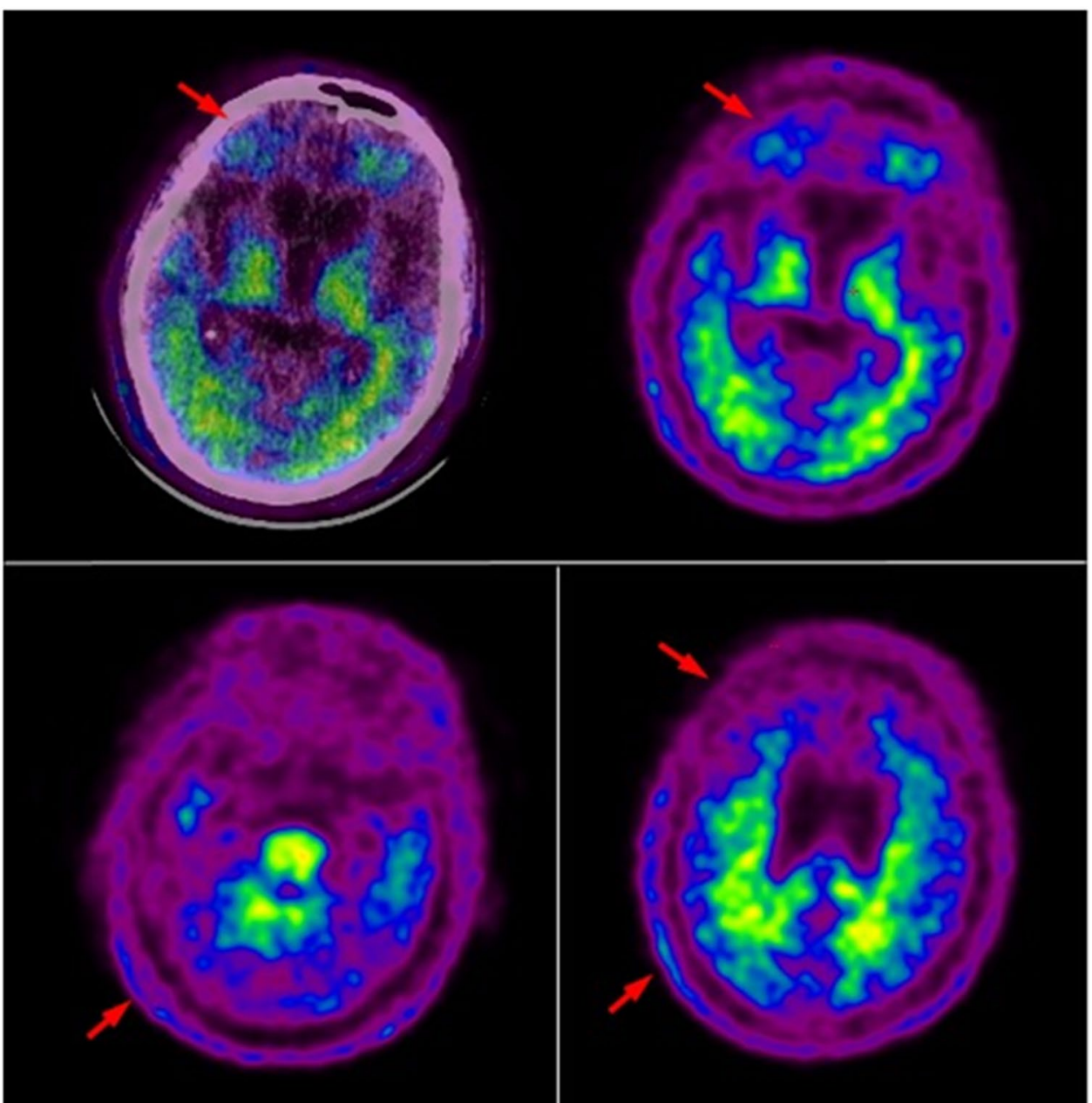

present but not corresponding to SWI hemorrhages. Interestingly in this case, the uptake is slightly higher in the left side, presenting hemorrhages. In these cases, the take-home message is to examine a recent $\mathrm{T} 2 *$ or SWI MR sequence to avoid confusing $\mathrm{AD}$ with amyloid angiopathy.

\section{Case \#8: 'suspected non-Alzheimer pathology' (SNAP)}

A female patient in the 7th decade of life; right-handed and 13 years of education. At first evaluation, she complained of gradually onset, immediate memory deficit for 1 year. She was aware of the deficit which caused anxiety and an apparent continuous 'state of confusion'. General medical and neurological examinations were inconclusive. Everyday functioning was essentially preserved. IADL and BADL $=$ all main functions preserved. The ApoE genotype: $\varepsilon 2 / 3$. The Neuropsychological assessment was MMSE 27/30; Memory tests: Episodic memory (Babcock story) borderline; Free and Cued Selective Reminding Test (Grober Buschke): low values in immediate memory and delayed recall with borderline values after cueing; normal other functions. The patient was classified as a single-domain amnestic Mild Cognitive Impairment. An MRI scan was performed (Fig. 9 panel A: axial T1 and T2-weighted MRI images in sagittal view show significant atrophy of the right medial temporal lobe and moderate atrophy of the right temporal pole (red arrows). The patient was classified as suspected MCI due to AD. To further support this diagnosis, a brain 18F FDG PET/CT was performed (Fig. 9, panel B: PET show hypometabolism in the right medial temporal lobe (red arrows) and a mild asymmetry (right $<$ left) in the temporal lateral cortex).

Figure 10c reports results of whole-brain voxel-wise analysis concerning age-matched controls. In red color clusters of significant hypometabolism for controls in both medial temporal lobes. The red arrow shows a measurable but not significant reduction in precuneus/posterior cingulate metabolism with respect to controls. Given the brain FDG PET scan results, imaging findings were mainly insufficient to confirm MCI due to $\mathrm{AD}$ and the patients and a clinical and neuropsychological follow-up was performed 1 year later. Neuropsychological assessment highlighted a similar profile with further worsening of cueing in delayed recall. Given the increasing state of anxiety generated by the subjective insight into the 
Fig. 7 Case \#6-double skull phenomenon and asymmetries: the double skull phenomenon is clearly visible on the right side (Fig. 8; first and second rows). Asymmetric activity between opposite sides of the brain is clearly visible in the skull and gray matter of the temporal and frontal lobes (Fig. 8; first row). Misalignment between amyPET and CT images is mostly visible in orbitofrontal cortex (Fig. 8; second row)
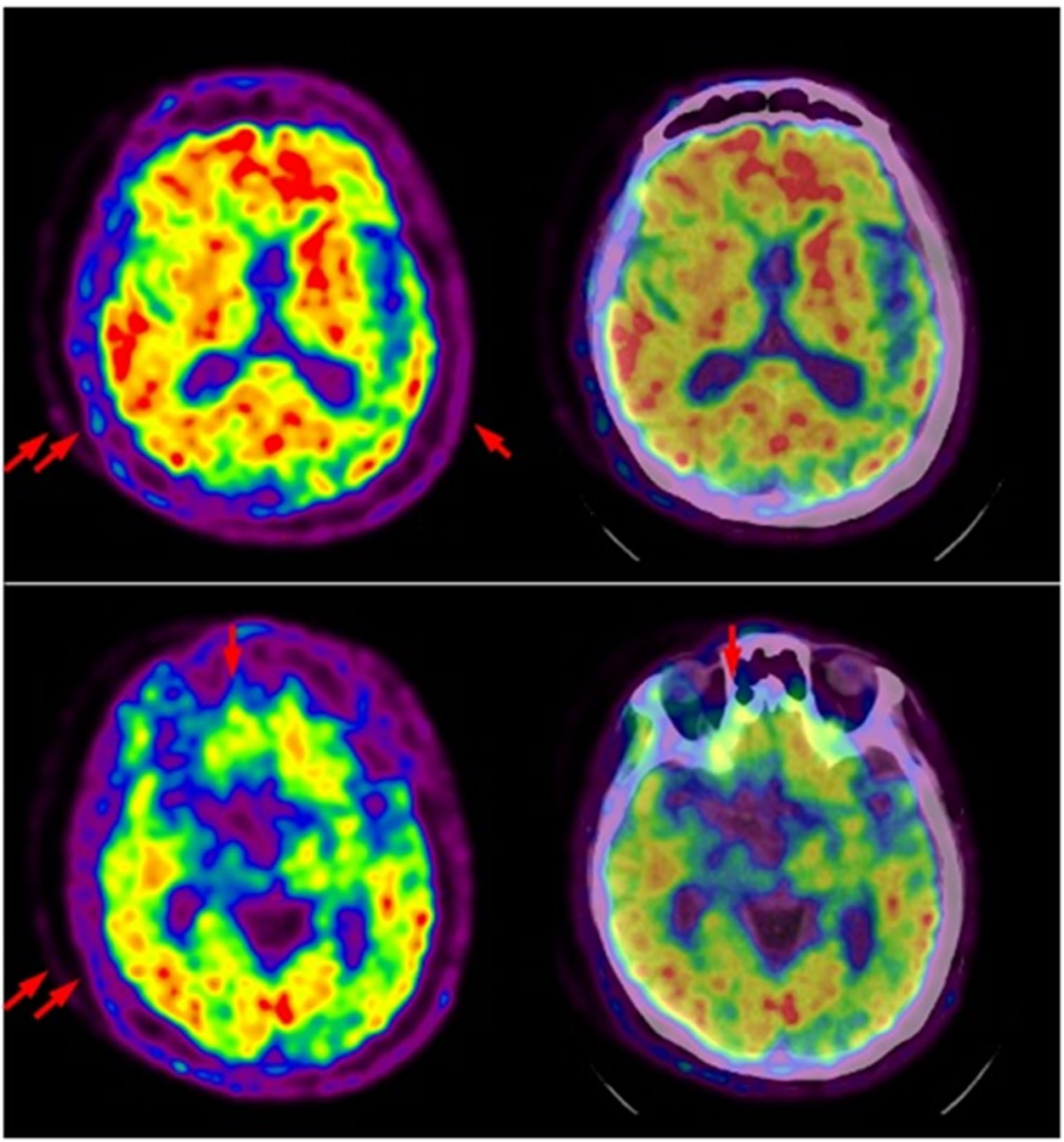

cognitive deficit, the patient was then submitted to an amyPET scan with 18F-Florbetapir (Fig. 10d: amy-PET scan) clearly negative for brain amyloidosis. The patient falls into the 'Suspected non Alzheimer Pathology', SNAP, category. SNAP is a biomarker-based concept denoting AD-like clinical presentation and evidence of neurodegeneration in individuals without brain amyloidosis [4, 19, 20]. In this case, given the prominent and relative isolated atrophy at medial temporal lobe level, the patient could be affected by hippocampal sclerosis or PART (primary age-related tauopathy). SNAP subjects progress to MCI or dementia more slowly and less frequently than patients $\mathrm{A}+\mathrm{N}+$ but more quickly than patients $\mathrm{A}-\mathrm{N}$ similarly to patients $\mathrm{A}+\mathrm{N}$.

\section{Quantification and imaging analysis}

\section{Case \#9 added value of semi-quantification in a borderline amy-PET case}

A female patient in the 7th decade of life. Right-handed; 12 years of education; cognitive complaint for approximately 1 year; difficulties in organizing, planning, cooking; no behavioral disturbances; full daily-life autonomy. The neuropsychological assessment showed MMSE 27/30; memory impairment on verbal anterograde assessment and visuospatial assessment; no praxis, gnosis, or speech 


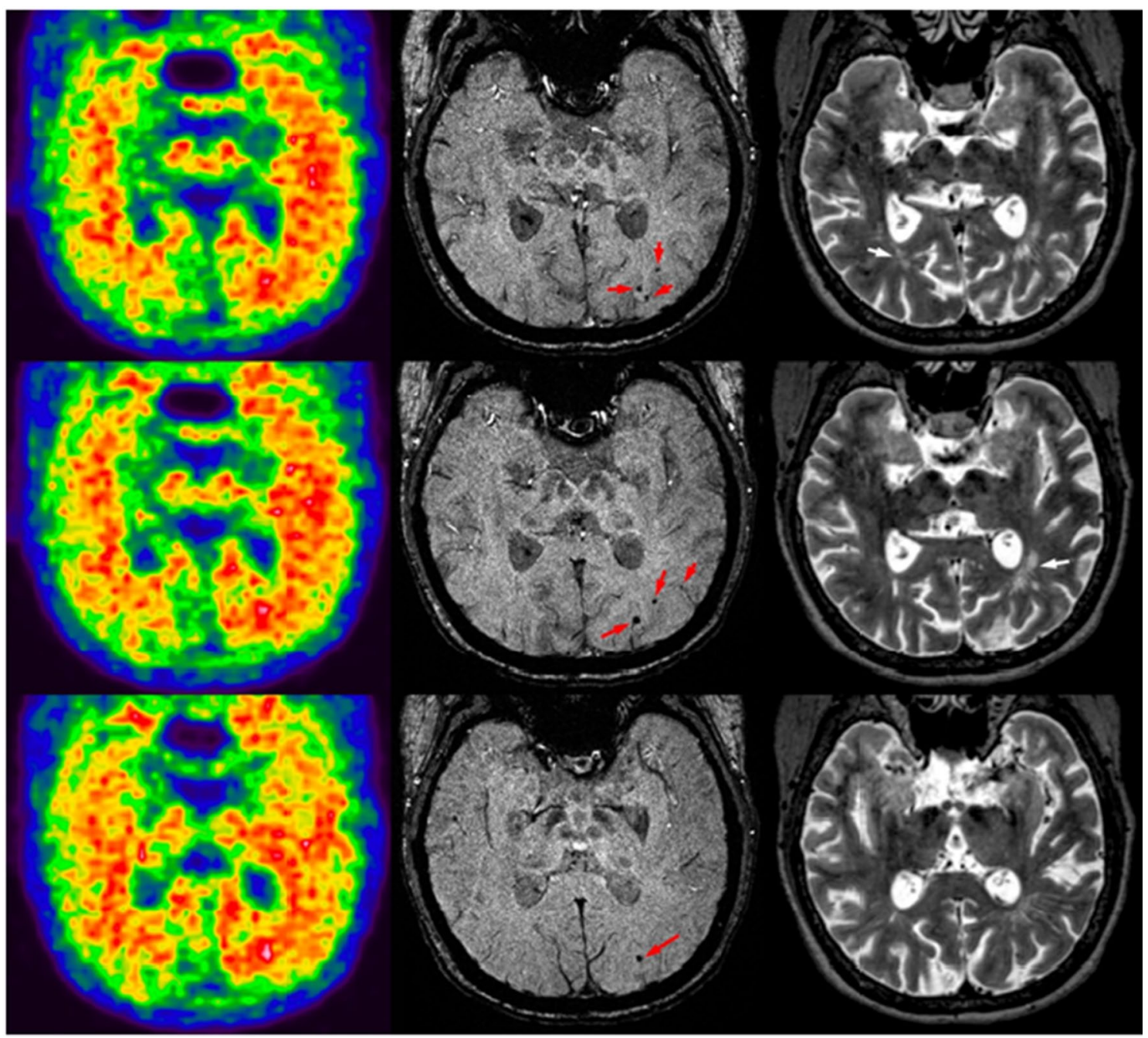

Fig. 8 Case \#7-positive amy-PET in a patient with cerebral amyloid angiopathy: amy-PET (left column) demonstrated a clear uptake of 18F-Florbetaben throughout the entire brain cortex consistent with AD. However, at susceptibility-weighted imaging (SWI), several little, ovoid, cortico-sub-cortical areas of hemorrhage (red arrows) have

impairment. The patient is labeled as amnestic MCI. MRI was performed (Fig. 10a, T2-weighted images) and it demonstrated multiple, small white matter hyperintensities in bilateral frontal regions. Mild atrophy of frontal lobes with very mild (grade I Scheltens's scale) left hippocampal atrophy.

Given the suspected of an MCI due AD, the patient is submitted to a brain 18F FDG PET scan (Fig. 10b). PET images show a bilateral moderate reduction of tracer uptake in the medial temporal lobes and the left posterior parietal cortex. Asymmetry (right $<$ left) also seems to be present in the anterior part of the temporal lateral cortex. 18F FDG PET findings were thus inconclusive to accurately support the presence of an MCI due to $\mathrm{AD}$ and a clinical follow-up was started. been noticed on the left parieto-temporo-occipital cortex that could be consistent with the diagnosis of cerebral amyloid angiopathy. Several T2 TSE hyperintensities in the white matter (white arrows on the right column) were also present but not corresponding to SWI hemorrhages

Two years later due to a further decline in her memory function, the evaluation of a second biomarker was proposed to assess the presence of brain amyloidosis. She was reluctant to perform a lumbar puncture so an amy-PET with 18F-Florbetapir was acquired with dual point technique in the framework a prospective research study. Early perfusion images acquired in the first 5 min after tracer administration substantially confirm findings previously evident on FDG PET (Fig. 10c). In fact, moderate hypoperfusion was evident in medial temporal lobe mainly on the left side. Moderate bilateral posterior parietal hypoperfusion was also evident.

Late (standard) amy-PET images acquired $30 \mathrm{~min}$ after tracer injection showed a possible loss of contrast between white and gray matter tracer uptake in both frontal lobes (Fig. 10d). A less clear reduction of contrast between white 
A

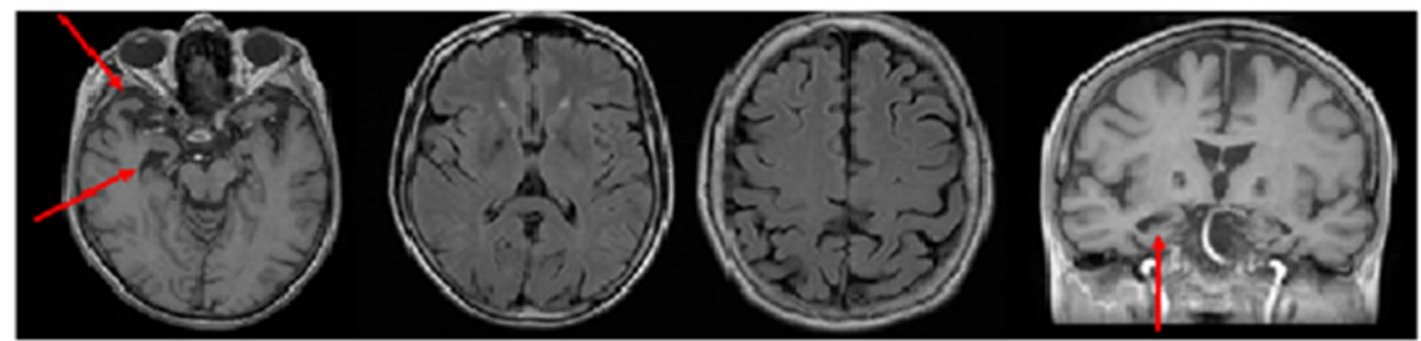

B
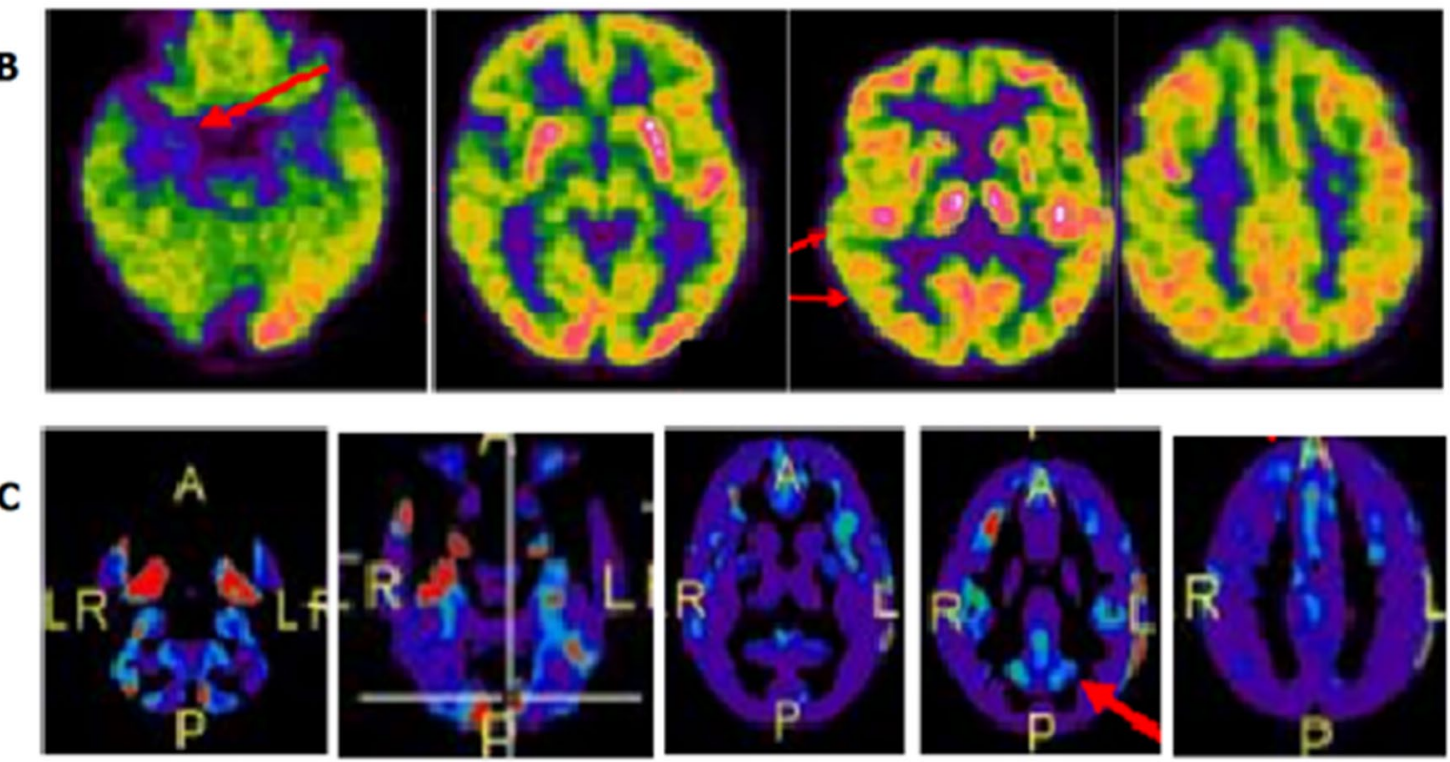

D
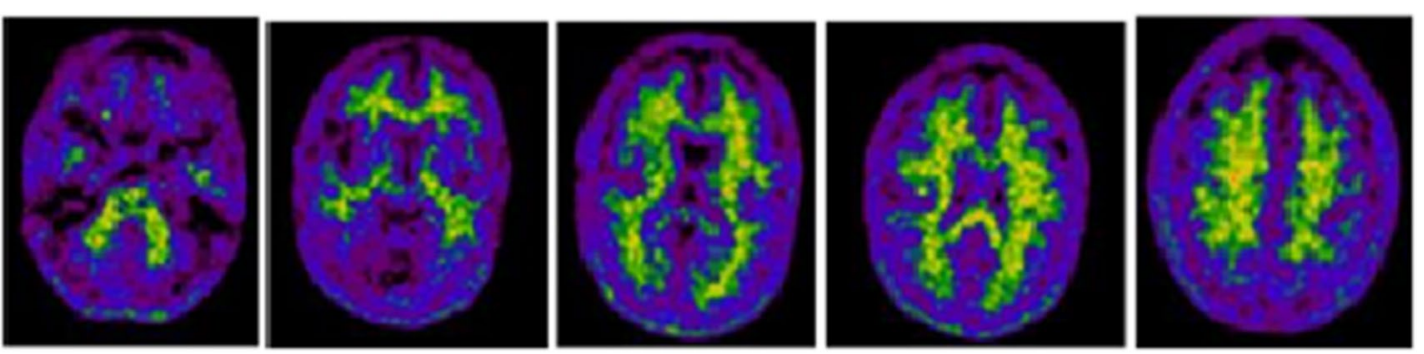

Fig. 9 Case \#8-'suspected non-Alzheimer pathology' (SNAP): MRI scan (a axial T1 and T2- weighted MRI images in sagittal view show significant atrophy of the right medial temporal lobe and moderate atrophy of the right temporal pole (red arrows). Given the clinical presentation and MRI findings, the patient was classified as suspected MCI due to AD. A brain 18F FDG PET/CT was performed (b PET show hypometabolism in the right medial temporal lobe (red arrows) and a mild asymmetry (right $<$ left) in temporal lateral cortex. c Reports results of whole-brain voxel-wise analysis with respect

and gray matter tracer uptake in both parietal lobes was also evident. However, the quality of these images was not high as the patient slightly moved between CT and PET acquisition and the loss of contrast (especially at frontal lobe level might be partially due to this motion artifact). Images was visually reported as a borderline/positive scan. Semi-quantification was performed to support visual reading. The semi-quantification values of the patient (SUVr, ELBA and TDr reported in Fig. 10e) are mapped onto the to age-matched controls. In red color clusters of significant hypometabolism concerning controls in both medial temporal lobes. The red arrow shows a measurable but not significant reduction in precuneus/ posterior cingulate metabolism concerning controls. d Amy-PET scan was clearly negative for brain amyloidosis. A preserved contrast is highlighted between the expected non-specific uptake in white matter and the uptake in the white matter. Gray matter uptake is always below the white matter uptake

normative curves, which trace the probability of belonging to a negative $(-1)$ or positive $(+1)$ cohort. These curves are traced for each of the 25 contralateral cortical ROI (dots, thin lines) and for the whole brain (square, thick line). The regional distribution values encompass both the negative and the positive cohorts, showing that this case is not a clear-cut positive but hinges on the borderline. However, all methods are in agreement, favoring a mild positive outcome. 
A

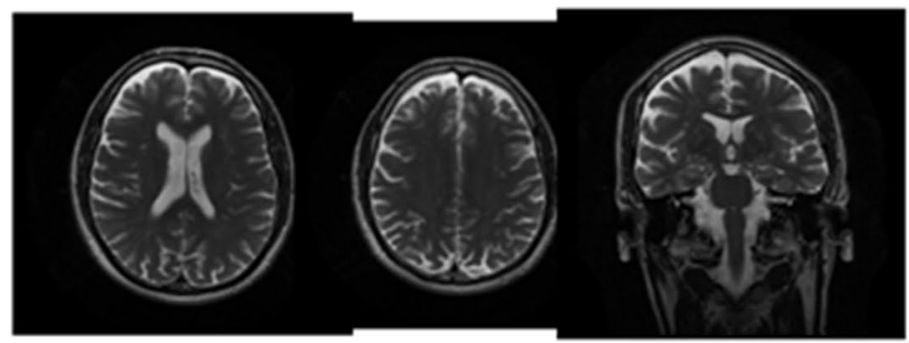

B
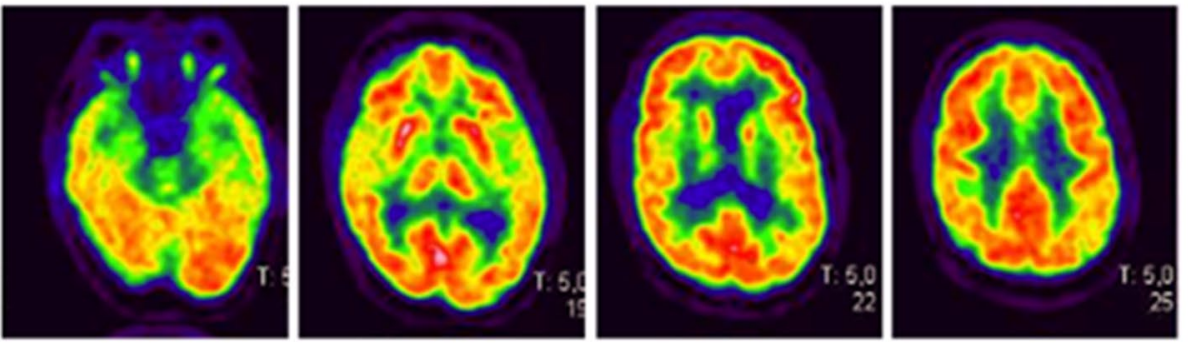

C

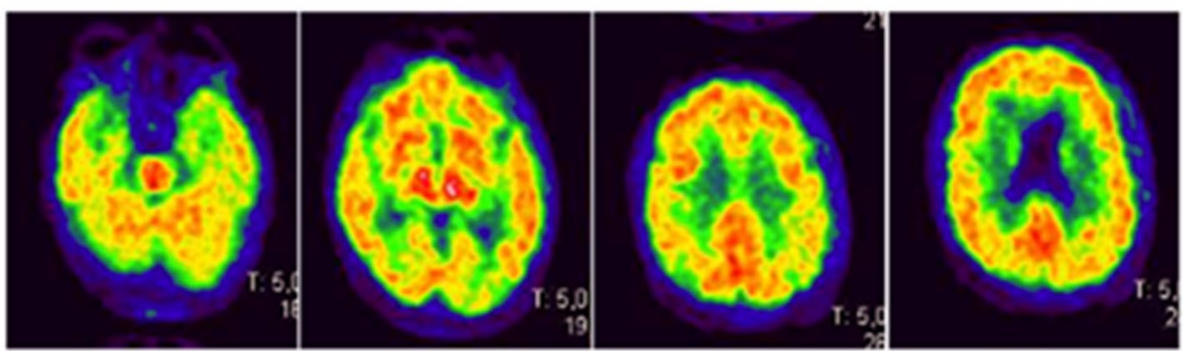

D

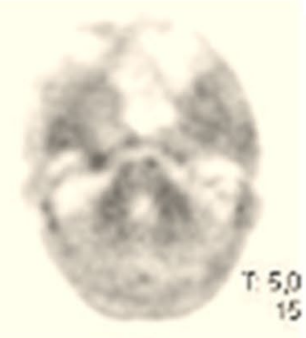

SUVr Plst

$\mathbf{E}$

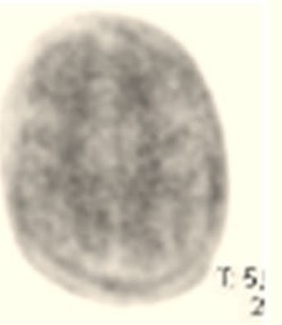

2.

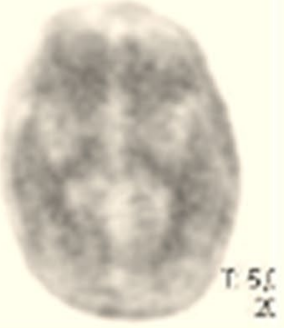

is

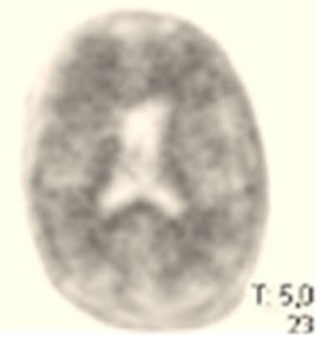

TDR Plot

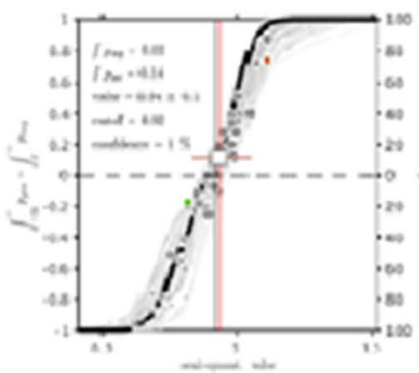

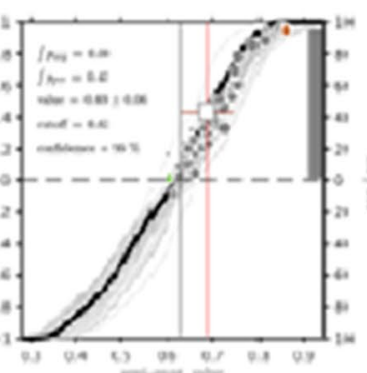

Elba Plot

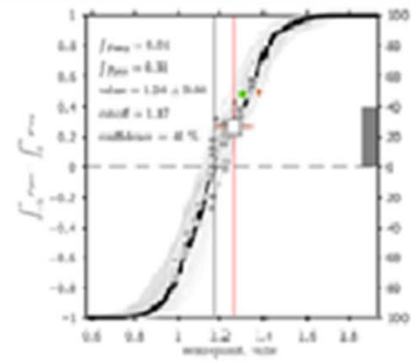

Fig. 10 Case \#9_added value of semi-quantification in a borderline amy-PET case: a MRI, T2-weighted images, b 18F FDG PET scan, $\mathbf{c}$ early perfusion 18F FDG PET scan, $\mathbf{d}$ amy-PET images, e SUVr, ELBA and TDr reported

\section{Discussion}

The recent development of amyloid targeting PET radiopharmaceuticals agents opens the possibility to measure in vivo pathogenic processes implicated in Alzheimer disease (AD) and has the potential to detect disease processes at the earliest stages with a potential role in the differential 
diagnosis and in interventional clinical trials aiming to develop new effective drugs $[21,22]$.

Analyzing brain diseases and its identification has remained a complex task for all diagnostic methods. Many conventional approaches were used for classification and identification tasks. The most common method associated with analyzing the brain disease is the vision-based approach practiced by the medical specialists which is still used very often. However, analyzing accurate results mostly depends on the ability of medical specialists. New artificial intelligence using machine-learning and deep-learning methods are emerging with the aim to improve the accuracy of detection process but the application on neurodegenerative disease and PET imaging is still limited due to the complex architecture and complex radiopharmaceuticals distribution [23].

The present paper aims to translate the theoretical knowledge of PET with available amyloid radioligands in the clinical routine. All possible scenarios are discussed providing the meaning of negative to positive amyloid images in different clinical settings, during early or late onset disease presentation, as well as of possible artifacts or pitfalls that may occur in the clinical practice using amy-PET. Furthermore, the role of visual analysis and semi-quantification approaches has been evaluated theoretically and practically on reliable clinical cases.

A detailed clinical approach is mandatory for the interpretation of images results. In our case \#1, the patient was rather young and presented only with few clinical symptoms of neurodegenerative disease, including amnestic MCI-single Domain, MMSE 28/30, family history of AD, anxiousdepressive disorder and inconclusive MRI scans. According to guidelines [15], the FDG PET was first planned to detect metabolic changes due to AD. However, the scan showed a metabolic pattern which was not wholly consistent with MCI due to AD because of normal glucose metabolism in posterior cingulate cortex and precuneus. Therefore, an amy-PET was required to confirm such a hypothesis and to rule out the diagnosis of AD. For this purpose, amy-PET was used to detect $\beta$-amyloid neuritic plaques in the brain. The visual approach has been recommended to include a co-registered CT to consider potential artifacts due to brain atrophy. Indeed, atrophy may be present in many areas of the brain and may render image interpretation more difficult as loss of gray matter will result in reduced tracer uptake making a positive scan more challenging to recognize. Besides, it is recommended that visual analysis of amy-PET images should be performed using specific color scales. In this case, the interpretation of amy-PET was performed using a Spectrum scale, but alternative color scales such as Sokoloff or Rainbow can be also used. Fourth, according to the manufacturer's instructions, including quality checks for the quantitation process, amy-PET images should be first interpreted visually and thereafter results of visual analysis results should be confirmed by quantitation approach. In our case, there was consistency between results of visual and quantification approach, confirming the presence of a negative scan. Finally, it has been highlighted in the case \#1 and case \#2 that a negative (as well as a positive) amy-PET scan gives an information of bio-molecular level concerning the absence (or the presence) of $\beta$-amyloid neuritic plaques in the brain and does not provide a diagnosis of a neurodegenerative disease per se. Therefore, it has been recommended that only such bio-molecular information should be included in the final report.

The well-defined negative predictive value of amy-PET has been confirmed giving an essential role of this tool in the differentiation of AD vs non-AD [7]. Regarding the positivity of amy-PET images, the case \#3 and case \#4 confirmed the good specificity also for the definition of typical and early AD presentation, particularly useful in case of clinical and/or CSF discrepancies (24). However, no significant results in amyloid plaque density and distribution of region of positivity were defined with similar presentation between typical and early AD at visual assessment.

Another critical issue in the evaluation of amy-PET recognizes possible artifacts as frequently occur in not collaborative patients due to significant cognitive impairment. Case \#5 and case \#6 presented in this pictorial issue showed two amy-PET artifacts due to patient movement during PET/CT acquisition, suggesting the check skull activity in search of motion and attenuation correction artifacts before examining gray matter tracer activity.

Other potential fonts of errors can be represented by false positivity in rare diseases such as amyloid angiopathy, as defined in case \#7. This case enhanced the clinical needing of MRI before the evaluation of AMY-PET images, confirming the value of T2* or SWI MR sequence to avoid confusing AD with amyloid angiopathy. Finally, in case \#8, the capability of amy-PET to clarify complex clinical presentation. The biological complexity of the disease Spectrum entering the differential diagnosis of dementing disorders is discussed in the framework of SNAP's case proven to have AD-like neurodegeneration on MRI or FDG PET or both but with a negative amy-PET.

As described in case \#9, semi-quantification analysis was fundamental for the differential diagnosis of borderline cases. Finally, as defined in the case \#9, the quantification of amy-PET images permits to evaluate difficult clinical conditions as for SNAP patients, characterized by AD-like clinical presentation and evidence of neurodegeneration in individuals without brain amyloidosis.

Funding All the authors declare that they did not receive any financial support for this study. 


\section{Declarations}

Ethical approval All the procedures performed in studies involving human participants were following ethical standards of the institutional and/or national research committee and with the 1964 Helsinki Declaration and its later amendments or comparable ethical standards.

Conflict of interest All the authors declare that they have no conflict of interest.

Informed consent Informed consent was obtained from all individual participants included in the study.

\section{References}

1. Dubois B, Feldman HH, Jacova C, Hampel H, Molinuevo JL, Blennow K et al (2014) Advancing research diagnostic criteria for Alzheimer's disease: the IWG-2 criteria. Lancet Neurol 13(6):614-629

2. McKhann GM, Knopman DS, Chertkow H, Hyman BT, Jack CR, Kawas CH et al (2011) The diagnosis of dementia due to Alzheimer's disease: recommendations from the National Institute on Aging-Alzheimer's Association workgroups on diagnostic guidelines for Alzheimer's disease. Alzheimers Dement 7(3):263-269

3. Albert MS, DeKosky ST, Dickson D, Dubois B, Feldman HH, Fox NC et al (2011) The diagnosis of mild cognitive impairment due to Alzheimer's disease: recommendations from the National Institute on Aging-Alzheimer's Association workgroups on diagnostic guidelines for Alzheimer's disease. Alzheimers Dement 7(3):270-279

4. Jack CR, Bennett DA, Blennow K, Carrillo MC, Dunn B, Haeberlein SB et al (2018) NIA-AA research framework: toward a biological definition of Alzheimer's disease. Alzheimer's Dement 14:535-562

5. Chiotis K, Saint-Aubert L, Boccardi M, Gietl A, Picco A, Varrone A et al (2017) Clinical validity of increased cortical uptake of amyloid ligands on PET as a biomarker for Alzheimer's disease in the context of a structured 5-phase development framework. Neurobiol Aging 52:214-227

6. Iaccarino L, Chiotis $\mathrm{K}$, Alongi $\mathrm{P}$, Almkvist $\mathrm{O}$, Wall A, Cerami $\mathrm{C}$ et al (2017) A cross-validation of FDG-and amyloid-PET biomarkers in mild cognitive impairment for the risk prediction to dementia due to Alzheimer's disease in a clinical setting. J Alzheimer's Dis 59(2):603-614

7. Morris E, Chalkidou A, Hammers A, Peacock J, Summers J, Keevil S (2016) Diagnostic accuracy of (18)F amyloid PET tracers for the diagnosis of Alzheimer's disease: a systematic review and meta-analysis. Eur J Nucl Med Mol Imaging 43(2):374-385

8. Shea YF, Barker W, Greig-Gusto MT, Loewenstein DA, Duara $\mathrm{R}$, Dekosky ST (2018) Impact of amyloid PET imaging in the memory clinic: a systematic review and meta-analysis. J Alzheimer's Dis 64(1):323-335

9. Chincarini A, Peira E, Corosu M, Morbelli S, Bauckneht M, Capitanio S et al (2020) A kinetics-based approach to amyloid PET semi-quantification. Eur J Nucl Med Mol Imaging 47(9):2175-2185

10. Chincarini A, Peira E, Morbelli S, Pardini M, Bauckneht M, Arbizu J et al (2019) Semi-quantification and grading of amyloid
PET: a project of the European Alzheimer's Disease Consortium (EADC). NeuroImage Clin 23:101846

11. Alongi P, Sardina DS, Coppola R, Scalisi S, Puglisi V, Arnone A et al (2019) 18F-Florbetaben PET/CT to assess Alzheimer's Disease: a new analysis method for regional amyloid quantification. J Neuroimaging 29(3):383-393

12. Boccardi M, Nicolosi V, Festari C, Bianchetti A, Cappa S, Chiasserini D et al (2020) Italian consensus recommendations for a biomarker-based aetiological diagnosis in mild cognitive impairment patients. Eur J Neurol 27(3):475-483

13. Chételat G, Arbizu J, Barthel H, Garibotto V, Law I, Morbelli S et al (2020) Amyloid-PET and 18F-FDG-PET in the diagnostic investigation of Alzheimer's disease and other dementias. Lancet Neurol 19:951-962

14. Minoshima S, Drzezga AE, Barthel H, Bohnen N, Djekidel M, Lewis DH et al (2016) SNMMI procedure standard/EANM practice guideline for amyloid PET imaging of the brain 10. J Nucl Med 57(8):1316-1322

15. Chincarini A, Sensi F, Rei L, Bossert I, Morbelli S, Guerra UP et al (2016) Standardized uptake value ratio-independent evaluation of brain amyloidosis. J Alzheimer's Dis. 54(4):1437-1457

16. Collij LE, Salvadó G, Shekari M, Alves IL, Reimand J, Wink AM et al (2021) Visual assessment of [18F]flutemetamol PET images can detect early amyloid pathology and grade its extent. Eur J Nucl Med Mol Imaging 48(7):2169-2182

17. Peira E, Grazzini M, Bauckneht M, Sensi F, Bosco P, Arnaldi D et al (2021) Probing the role of a regional quantitative assessment of amyloid PET. J Alzheimer's Dis 80(1):383-396

18. Presotto L, Iaccarino L, Sala A, Vanoli EG, Muscio C, Nigri A et al (2018) Low-dose CT for the spatial normalization of PET images: a validation procedure for amyloid-PET semi-quantification. NeuroImage Clin 20:153-160

19. Burnham SC, Bourgeat P, Doré V, Savage G, Brown B, Laws $S$ et al (2016) Clinical and cognitive trajectories in cognitively healthy elderly individuals with suspected non-Alzheimer's disease pathophysiology (SNAP) or Alzheimer's disease pathology: a longitudinal study. Lancet Neurol 15(10):1044-1053

20. Dani M, Brooks DJ, Edison P (2017) Suspected non Alzheimer's pathology - is it non-Alzheimer's or non-amyloid? Ageing Res Rev 36:20-31

21. Mattsson N, Carrillo MC, Dean RA, Devous MD, Nikolcheva T, Pesini P et al (2015) Revolutionizing Alzheimer's disease and clinical trials through biomarkers. Alzheimer's Dement Diagn Assess Dis Monit 1:412-419

22. Leuzy A, Savitcheva I, Chiotis K, Lilja J, Andersen P, Bogdanovic $\mathrm{N}$ et al (2019) Clinical impact of [18 F]flutemetamol PET among memory clinic patients with an unclear diagnosis. Eur J Nucl Med Mol Imaging 46(6):1276-1286

23. Haq EU, Huang J, Kang L, Haq HU, Zhan T (2020) Image-based state-of-the-art techniques for the identification and classification of brain diseases: a review. Med Biol Eng Comput 58:2603-2620

24. Ramusino MC, Garibotto V, Bacchin R, Altomare D, Dodich A, Assal $F$ et al (2020) Incremental value of amyloid-PET versus CSF in the diagnosis of Alzheimer's disease. Eur J Nucl Med Mol Imaging. 47(2):270-280

Publisher's Note Springer Nature remains neutral with regard to jurisdictional claims in published maps and institutional affiliations. 